Pacific Journal of Mathematic

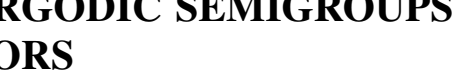




\section{INVARIANT MEASURES FOR ERGODIC SEMIGROUPS OF OPERATORS}

\section{RYOTARO SATO}

In this paper the invariant measure problem is investigated for ergodic semigroups (in the sense of Eberlein) of positive linear operators on the $L_{1}$-space of a probability measure space. Various results in operator ergodic theory are generalized and extended to give a unified approach to the problem. The main step in this approach is the following result: There exists a positive linear functional $\varphi$ on the space $B(\Lambda)$ of all bounded real valued functions on a directed set $\Lambda$ such that

$$
\liminf _{\alpha \in A} \xi(\alpha) \leqq \varphi(\xi) \leqq \lim _{\alpha \in A} \sup \xi(\alpha)
$$

for all $\xi \in B(\Lambda)$.

Let $(X, \mathfrak{M}, m)$ be a probability measure space and let $L_{p}(X)=$ $L_{p}(X, \mathfrak{M}, m), 1 \leqq p \leqq \infty$, be the Banach spaces defined as usual with respect to $(X, \mathfrak{M}, m)$. For a set $A \in \mathfrak{M}, 1_{A}$ denotes the indicator function of $A$ and $L_{p}(A)$ denotes the Banach space of all $L_{p}(X)$ functions that vanish a.e. on $X-A$. If $f \in L_{p}(X)$, we define supp $f$ to be the set of all $x$ in $X$ at which $f(x) \neq 0$. Relations introduced below are assumed to hold modulo sets of $m$-measure zero.

Let $\Sigma=\{T\}$ be a semigroup of positive linear operators on $L_{1}(X)$. A function $f \in L_{1}(X)$ is called $\Sigma$-fixed if $T f=f$ for every $T \in \Sigma$. The problem of finding necessary and sufficient conditions for the existence of a $\Sigma$-fixed $f_{0} \in L_{1}(X)$, with $f_{0}>0$ a.e. on $X$, has been studied by many authors (see, for example, [4], [5], [8], [9], [11], [12], [13], [14], [17], [18], [21], [22], [23], [24], [25], [27], and others). In the present paper we intend to investigate the problem for ergodic semigroups $\Sigma$ in the sense of Eberlein, and generalize and extend various known results to give a unified approach to the problem.

For $f \in L_{1}(X)$, we denote by $\overline{c o} \Sigma f$ the closed convex hull of the set $\{T f: T \in \Sigma\} . \quad \Sigma$ is said to be left [resp. right] ergodic if there exists a net $\left(T_{\alpha}, \alpha \in \Lambda\right)$ of positive linear operators on $L_{1}(X)$ satisfying

(a) $\lim \sup _{\alpha}\left\|T_{\alpha}\right\|<\infty$,

(b) for every $f \in L_{1}(X)$ and every $\alpha \in \Lambda, T_{\alpha} f \in \overline{\mathrm{co}} \Sigma f$,

(c) for every $T \in \Sigma, \lim _{\alpha} T T_{\alpha}-T_{\alpha} T=0$ [resp. $\lim _{\alpha} T_{\alpha}-T_{\alpha}=0$ ],

where the convergence can be either in the uniform, strong, or weak operator topology. (Cf. Eberlein [7] and Day [3].) The above net $\left(T_{\alpha}, \alpha \in \Lambda\right)$ is said to be left [resp. right] $\Sigma$-ergodic. If $\left(T_{\alpha}, \alpha \in \Lambda\right)$ is 
both left and right $\Sigma$-ergodic, then it is said, simply, to be $\Sigma$-ergodic. If $\Sigma$ has a $\Sigma$-ergodic net, then $\Sigma$ is said to be ergodic.

In the next section we investigate the invariant measure problem for weakly left ergodic semigroups $\Sigma$, by making use of the fact that if $\Lambda$ is a directed set, then there exists a positive linear functional $\phi$ on the space $B(\Lambda)$ of all bounded real valued functions on $\Lambda$ satisfying

$$
\lim _{\alpha} \inf \xi(\alpha) \leqq \varphi(\xi) \leqq \lim _{\alpha} \sup \xi(\alpha)
$$

for all $\xi \in B(\Lambda)$. Theorem 1 states that if $\left(T_{\alpha}, \alpha \in \Lambda\right)$ is a weakly left $\Sigma$-ergodic net, then there exists a $\Sigma$-fixed $f_{0} \in L_{1}(X)$, with $f_{0}>0$ a.e. on $X$, if and only if $A \in \mathfrak{M}$ and $m(A)>0$ imply

$$
\lim _{\alpha} \inf \int_{A} T_{\alpha} 1 d m>0 \text {. }
$$

Theorem 2, together with Theorem 1 , asserts that if $\Sigma$ is a weakly left ergodic semigroup of positive linear contraction operators on $L_{1}(X)$, then there exists a $\Sigma$-fixed $f_{0} \in L_{1}(X)$, with $f_{0}>0$ a.e. on $X$, if and only if $0 \leqq u \in L_{\infty}(X)$ and $\sum_{n=1}^{\infty} T_{n}^{*} u \in L_{\infty}(X)$ for some sequence $\left(T_{n}, n \geqq 1\right)$ in $\Sigma$ imply $u=0$ a.e. on $X$; in particular, if $\Sigma$ has a uniformly left $\Sigma$-ergodic net $\left(T_{\alpha}, \alpha \in \Lambda\right)$, then there exists a $\Sigma$-fixed $f_{0} \in L_{1}(X)$, with $f_{0}>0$ a.e. on $X$, if and only if $A \in \mathfrak{M}$ and $m(A)>0$ imply $\lim \sup _{\alpha}\left\|T_{\alpha}^{*} 1_{A}\right\|_{\infty}>0$. Here it should be remarked that Theorems 1 and 2 include results due to Dean and Sucheston [4], Foguel [8], Lin [14], Neveu [17], [18], and Sachdeva [21], for every (left) amenable semigroup of uniformly bounded linear operators on $L_{1}(X)$ is uniformly (left) ergodic (see Day [3]).

In the third section we investigate the problem for weakly or uniformly ergodic semigroups $\Sigma$ and obtain two theorems, one of which states that if $\left(T_{\alpha}, \alpha \in \Lambda\right)$ is a uniformly $\Sigma$-ergodic net, then there exists a $\Sigma$-fixed $f_{0} \in L_{1}(X)$, with $f_{0}>0$ a.e. on $X$, if and only if $A \in \mathfrak{M}$ and $m(A)>0$ imply $\lim \sup _{\alpha} T_{\alpha}^{*} 1_{A} \not \equiv 0$, where $\lim \sup _{\alpha} T_{\alpha}^{*} 1_{A}$ is defined by

$$
\lim \sup _{\alpha} T_{\alpha}^{*} 1_{A}=\underset{\alpha}{\operatorname{ess} \inf }\left(\underset{\beta>\alpha}{\operatorname{essup}} T_{\beta}^{*} 1_{A}\right)
$$

(cf. Neveu [16], Proposition II.4.1, p. 44).

In the last section we apply these obtained results to explian some special examples. Among other things, we observe that if $T$ is a positive linear operator on $L_{1}(X)$ and $r$ is a positive real number such that

$$
\sup _{n \geqq 0}\left\|\sigma_{n}^{r}(T)\right\|<\infty,
$$


where $\sigma_{n}^{r}(T), n=0,1, \cdots$, denote the Cesàro means of order $r$ of the sequence $\left(T^{n}, n \geqq 0\right)$, then there exists an $f_{0} \in L_{1}(X)$, with $T f_{0}=f_{0}$ and $f_{0}>0$ a.e. on $X$, if and only if $A \in \mathfrak{M}$ and $m(A)>0$ imply

$$
\limsup _{n \rightarrow \infty} \sigma_{n}^{r}(T)^{*} 1_{A} \not \equiv 0 \text {. }
$$

This is a generalization of a result due to Derriennic and Lin [5], who considered only the case $\sup _{n \geqq 0}\left\|\sigma_{n}^{1}(T)\right\|<\infty$.

2. Invariant measures for left ergodic semigroups. In this section we investigate the invariant measure problem for weakly left ergodic semigroups $\Sigma=\{T\}$ of positive linear operators on $L_{1}(X)$. First of all we shall prove the following lemma, which is fundamental throughout the paper.

Lemma 1 (S. Banach). Let $\Lambda$ be a directed set and $B(\Lambda)$ the space of all bounded real valued functions on 1 . Then there exists a positive linear functional $\varnothing$ on $B(\Lambda)$ satisfying

$$
\lim _{\alpha} \inf \xi(\alpha) \leqq \varphi(\xi) \leqq \lim _{\alpha} \sup \xi(\alpha)
$$

for all $\xi \in B(\Lambda)$.

Proof. For $\alpha \in \Lambda$, let $\delta_{\alpha}$ denote the linear functional on $B(\Lambda)$ defined by $\delta_{\alpha}(\xi)=\xi(\alpha)$ for all $\xi \in B(\Lambda)$. Since $\left\|\delta_{\alpha}\right\|=1$ for all $\alpha \in \Lambda$, the Banach-Alaoglu theorem (see, for example, Rudin [20], p. 66) shows that there exists a linear functional $\phi$ on $B(\Lambda)$ which is a weak-star cluster point of the net $\left(\delta_{\alpha}, \alpha \in \Lambda\right)$ in $B(\Lambda)^{*}$, the dual space of $B(\Lambda)$. It is clear that $\varnothing$ satisfies (1) for all $\xi \in B(\Lambda)$.

For a directed set $\Lambda$, let GL $(\Lambda)$ denote the set of all $\varphi \in B(\Lambda)^{*}$ that satisfy (1) for all $\xi \in B(\Lambda)$. Since $\mathrm{GL}(\Lambda)$ is not empty by the above lemma, we can define a functional $\Delta$ on $B(\Lambda)$ by the relation:

$$
\Delta(\xi)=\sup \{\varphi(\xi): \varphi \in \mathrm{GL}(\Lambda)\} \quad(\xi \in B(\Lambda)) .
$$

It follows from a slight modification of the proof of Lemma 1 that if $\Lambda$ is a countable directed set, then

$$
\Delta(\xi)=\lim \sup _{\alpha} \xi(\alpha) \text { for all } \xi \in B(\Lambda) .
$$

From now on let us always assume that $\Sigma=\{T\}$ is a fixed semigroup of positive linear operators on $L_{1}(X)$. The boundedness of $\Sigma$ is not assumed, unless the contrary is explicitly specified.

THEOREM 1. If $\left(T_{\alpha}, \alpha \in \Lambda\right)$ is a weakly left $\Sigma$-ergodic net, then the following conditions are equivalent: 
(0) There exests a $\Sigma$-fixed $f_{0} \in L_{1}(X)$ with $f_{0}>0$ a.e. on $X$.

(i) $A \in \mathfrak{M}$ and $m(A)>0$ imply $\lim \inf _{\alpha} \int_{A} T_{\alpha} 1 d m>0$.

Proof. Choose a $\beta \in \Lambda$ and a constant $K$ such that

$$
\left\|T_{\alpha}\right\| \leqq K \text { for all } \alpha \geqq \beta \text {. }
$$

Then the proof proceeds as follows.

$(0) \Rightarrow(\mathrm{i})$ : For each positive integer $n$, define

$$
h_{n}(x)=\min \left\{f_{0}(x), n\right\} \quad(x \in X) .
$$

Then $\lim _{n \rightarrow \infty}\left\|f_{0}-h_{n}\right\|_{1}=0$, and since $f_{0}>0$ a.e. on $X$, it follows that, for any $A \in \mathfrak{M}$ with $m(A)>0$,

$$
(0<) \int_{A} f_{0} d m=\int_{A} T_{\alpha} f_{0} d m=\int_{A} T_{\alpha} h_{n} d m+\int_{A} T_{\alpha}\left(f_{0}-h_{n}\right) d m,
$$

and that

$$
\lim _{n \rightarrow \infty}\left(\sup _{\alpha \geq \beta} \int_{A} T_{\alpha}\left(f_{0}-h_{n}\right) d m\right) \leqq \lim _{n \rightarrow \infty} K\left\|f_{0}-h_{n}\right\|_{1}=0 .
$$

Therefore, for a sufficiently large $n$, we have

$$
n \int_{A} T_{\alpha} 1 d m \geqq \int_{A} T_{\alpha} h_{n} d m \geqq \int_{A} f_{0} d m-K\left\|f_{0}-h_{n}\right\|_{1}>0
$$

for all $\alpha \geqq \beta$, and hence $\lim \inf _{\alpha} \int_{A} T_{\alpha} 1 d m>0$.

(i) $\Rightarrow(0)$ : Let $\varphi \in \mathrm{GL}(\Lambda)$, and define a positive linear functional $\mu$ on $L_{\infty}(X)$ by the relation:

$$
\langle\mu, f\rangle=\varphi\left(\left\langle T_{\alpha} 1, f\right\rangle\right) \quad\left(f \in L_{\infty}(X)\right),
$$

where $\left\langle T_{\alpha} 1, f\right\rangle=\int\left(T_{\alpha} 1\right) f d m$. Since, for each $T \in \Sigma$ and each $f \in L_{\infty}(X)^{\text {‘ }}$

$$
\begin{aligned}
\left\langle T^{* *} \mu, f\right\rangle & =\left\langle\mu, T^{*} f\right\rangle=\varphi\left(\left\langle T_{\alpha} 1, T^{*} f\right\rangle\right) \\
& =\varphi\left(\left\langle T T_{\alpha} 1, f\right\rangle\right)=\varphi\left(\left\langle T_{\alpha} 1, f\right\rangle\right)=\langle\mu, f\rangle,
\end{aligned}
$$

it follows that $T^{* *} \mu=\mu$ for all $T \in \Sigma$. Let $\mu_{0}$ denote the maximal (countably additive) measure, with $0 \leqq \mu_{0} \leqq \mu$ (cf. Neveu [18], Lemma 1), and let $g=d \mu_{0} / d m\left(\in L_{1}(X)\right)$. It then follows that $T g \leqq g$ for all $T \in \Sigma$, and thus if we define another linear functional $\lambda$ on $L_{\infty}(X)$ by the relation:

$$
\langle\lambda, f\rangle=\varphi\left(\left\langle T_{\alpha} g, f\right\rangle\right) \quad\left(f \in L_{\infty}(X)\right),
$$

then $\lambda$ is a (countably additive) measure absolutely continuous with respect to $m$, and $T^{* *} \lambda=\lambda$ for all $T \in \Sigma$. 
To see that $\lambda$ is equivalent to $m$, we note that (i) implies $\mu(A)>0$ for any $A \in \mathfrak{M}$ with $m(A)>0$. Hence it follows from Lemma 1 of [18] that $\mu_{0}$ is equivalent to $m$, and therefore $g>0$ a.e. on $X$. Putting, for each positive integer $n, g_{n}(x)=\min \{1, n g(x)\}(x \in X)$, we then obtain $\lim _{n \rightarrow \infty}\left\|1-g_{n}\right\|_{1}=0$. Let $A \in \mathfrak{M}$ with $m(A)>0$ be given, and choose an $\varepsilon>0$ and an $\alpha_{0} \in \Lambda$ so that $\alpha_{0} \geqq \beta$ and

$$
\int_{A} T_{\alpha} 1 d m>\varepsilon \text { for all } \alpha \geqq \alpha_{0} \text {. }
$$

Then, for a sufficiently large $n$, we have

$$
n \int_{A} T_{\alpha} g d m \geqq \int_{A} T_{\alpha} g_{n} d m>\varepsilon-K\left\|1-g_{n}\right\|_{1}>0
$$

for all $\alpha \geqq \alpha_{0}$, and therefore $\lim \inf _{\alpha} \int_{A} T_{\alpha} g d m>0$. This proves that $\lambda$ is equivalent to $m$.

The proof is complete.

THEOREM 2. Suppose $\|T\| \leqq 1$ for all $T \in \Sigma$. If $\left(T_{\alpha}, \alpha \in \Lambda\right)$ is a weakly left $\Sigma$-ergodic net, then the following conditions are equivalent:

(i ) $A \in \mathfrak{M}$ and $m(A)>0$ imply $\inf _{T \in \Sigma} \int_{A} T 1 d m>0$.

(ii) $A \in \mathfrak{M}$ and $m(A)>0$ imply $\Delta\left(\int_{A} T_{\alpha} 1 d m\right)>0$.

(iii) $0 \leqq u \in L_{\infty}(X)$ and $\sum_{n=1}^{\infty} T_{n}^{*} u \in L_{\infty}(X)$ for some sequence $\left(T_{n}, n \geqq 1\right)$ in $\Sigma$ imply $u=0$ a.e. on $X$.

In particular, if $\left(T_{\alpha}, \alpha \in \Lambda\right)$ is a uniformly left $\Sigma$-ergodic net, then the following condition and the above three conditions are still equivalent:

(iv) $A \in \mathfrak{M}$ and $m(A)>0$ imply $\lim \sup _{\alpha}\left\|T_{\alpha}^{*} 1_{A}\right\|_{\infty}>0$.

For the proof of this theorem we need the next two lemmas.

Lemma 2. Suppose $\|T\| \leqq 1$ for all $T \in \Sigma$. If $0 \leqq u \in L_{\infty}(X)$ satisfies $\inf \{\langle T 1, u\rangle: T \in \Sigma\}=0$, then there exists a nonnegative function $h$ in $L_{\infty}(X)$ and a sequence $\left(T_{n}, n \geqq 1\right)$ in $\Sigma$ such that

$$
\operatorname{supp} h=\operatorname{supp} u \text { and } \sum_{n=1}^{\infty} T_{n}^{*} h \in L_{\infty}(X) \text {. }
$$

LEMMA 3. Let $\left(T_{\alpha}, \alpha \in \Lambda\right)$ be a uniformly left L-ergodic net. If $0 \leqq u \in L_{\infty}(X)$ satisfies $\sum_{n=1}^{\infty} T_{n}^{*} u \in L_{\infty}(X)$ for some sequence $\left(T_{n}, n \geqq 1\right)$ in $\Sigma$, then $\lim _{\alpha}\left\|T_{\alpha}^{*} u\right\|_{\infty}=0$.

Proof of Lemma 2. This follows from a slight modification of 
the proof of (i) $\Rightarrow$ (iv) in Proposition 2 of the author [23] (see pp. 193-194 in [23]), and hence we omit the details.

Proof of Lemma 3. Given an $\varepsilon>0$, define

$$
E_{\alpha}=\left\{x \in X: T_{\alpha}^{*} u(x) \geqq \varepsilon\right\} \quad(\alpha \in \Lambda),
$$

and write

$$
\Gamma=\left\{\alpha \in \Lambda: m\left(E_{\alpha}\right)>0\right\} \text {. }
$$

If $\alpha \in \Gamma$, pick a nonnegative function $f_{\alpha} \in L_{1}(X)$, with supp $f_{\alpha} \subset E_{\alpha}$ and $\left\|f_{\alpha}\right\|_{1}=1$, and define $g_{\alpha}=T_{\alpha} f_{\alpha}$. Then we have

$$
g_{\alpha} \geqq 0, \quad\left\langle g_{\alpha}, u\right\rangle \geqq \varepsilon, \text { and } \limsup _{\alpha \in \Gamma}\left\|g_{\alpha}\right\|_{1}<\infty \text {. }
$$

We now prove that there exists an $\alpha_{0} \in \Lambda$ such that $\alpha \in \Gamma$ and $\alpha>\alpha_{0}$ implies $\alpha \in \Gamma$. Assume the contrary, and let $\mu$ denote a weak-star clustar point of the net $\left(g_{\alpha}, \alpha \in \Gamma\right)$ in $L_{\infty}(X)^{*}$. (Since $\lim \sup _{\alpha}\left\|g_{\alpha}\right\|_{1}<\infty$, there exists at least one such.) Then, for each $T \in \Sigma$ and each $f \in L_{\infty}(X)$, we have

$$
\begin{aligned}
\left|\left\langle T^{* *} \mu-\mu, f\right\rangle\right| \leqq & \left|\left\langle\mu-g_{\alpha}, T^{*} f\right\rangle\right|+\left|\left\langle T g_{\alpha}-g_{\alpha}, f\right\rangle\right| \\
& +\left|\left\langle g_{\alpha}-\mu, f\right\rangle\right|,
\end{aligned}
$$

and

$$
\lim _{\alpha \in \Gamma}\left|\left\langle T g_{\alpha}-g_{\alpha}, f\right\rangle\right| \leqq\|f\|_{\infty} \lim _{\alpha \in \Gamma}\left\|T T_{\alpha}-T_{\alpha}\right\|=0 .
$$

Moreover, since $\mu$ is a weak-star cluster point of the net $\left(g_{\alpha}, \alpha \in \Gamma\right)$, for any $\delta>0$ and any $\alpha \in \Gamma$ there exists a $\beta \in \Gamma$ such that $\beta \geqq \alpha$,

$$
\left|\left\langle\mu-g_{\beta}, T^{*} f\right\rangle\right|<\delta \text { and }\left|\left\langle\mu-g_{\beta}, f\right\rangle\right|<\delta .
$$

Hence it follows that $\left|\left\langle T^{* *} \mu-\mu, f\right\rangle\right|\langle 2 \delta$, and since $\delta$ was arbitrary, we see that $\left\langle T^{* *} \mu-\mu, f\right\rangle=0$. Therefore $T^{* *} \mu=\mu$ for all $T \in \Sigma$, and hence $\langle\mu, u\rangle=0$, because

$$
0 \leqq n\langle\mu, u\rangle=\left\langle\mu, \sum_{i=1}^{n} T_{i}^{*} u\right\rangle \leqq\left\langle\mu, \sum_{i=1}^{\infty} T_{i}^{*} u\right\rangle<\infty .
$$

But this is a contradiction, since $\left\langle g_{\alpha}, u\right\rangle \geqq \varepsilon$ for all $\alpha \in \Gamma$.

The proof is complete.

Proof of Theorem 2. If $\left(T_{\alpha}, \alpha \in \Lambda\right)$ is a weakly left $\Sigma$-ergodic net, then the implications (i) $\Rightarrow$ (ii) $\Rightarrow$ (iii) and (i) $\Rightarrow$ (iv) are immediate. The implication (iii) $\Rightarrow$ (i) is a direct consequence of Lemma 2. If $\left(T_{\alpha}, \alpha \in \Lambda\right)$ is a uniformly left $\Sigma$-ergodic net, then the implication (iv) $\Rightarrow$ (iii) follows from Lemma 3. 
The proof is complete.

The next theorem is a counterpart to Theorems 1 and 2.

THEOREM 3. Suppose $\|T\| \leqq 1$ for all $T \in \Sigma$. If $\left(T_{\alpha}, \alpha \in \Lambda\right)$ is a weakly left $\Sigma$-ergodic net, then the following conditions are equivalent:

(0) The only $g \in L_{1}(X)$ such that $T g=g$ for all $T \in \Sigma$ is 0 .

(i) There exists a function $u \in L_{\infty}(X)$ such that $u>0$ a.e. on. $X$ and $\inf _{T \in \Sigma} \int(T 1) u d m=0$.

(ii) There exists a function $u \in L_{\infty}(X)$ such that $u>0$ a.e. on $X$ and $\Delta\left(\int\left(T_{\alpha} 1\right) u d m\right)=0$.

(iii) There exists a function $u \in L_{\infty}(X)$ and a sequence $\left(T_{n}, n \geqq 1\right)$ in $\Sigma$ such that $u>0$ a.e. on $X$ and $\sum_{n=1}^{\infty} T_{n}^{*} u \in L_{\infty}(X)$.

In particular, if $\left(T_{\alpha}, \alpha \in \Lambda\right)$ is a uniformly left $\Sigma$-ergodic net, then the following condition and the above four conditions are still equivalent:

(iv) There exists a function $u \in L_{\infty}(X)$ such that $u>0$ a.e. on $X$ and $\lim _{\alpha}\left\|T_{\alpha}^{*} u\right\|_{\infty}=0$.

Proof. $(0) \Rightarrow(\mathrm{i})$ : As in the proof of $(\mathrm{i}) \Rightarrow(0)$ in Theorem 1 , for $\varphi \in \mathrm{GL}(\Lambda)$ define a positive linear functional $\mu$ on $L_{\infty}(X)$ by the relation:

$$
\langle\mu, f\rangle=\varphi\left(\left\langle T_{\alpha} 1, f\right\rangle\right) \quad\left(f \in L_{\infty}(X)\right) .
$$

Let $\mu_{0}$ denote the maximal (countably additive) measure with $0 \leqq$ $\mu_{0} \leqq \mu$. Then, for each $T \in \Sigma, T^{* *} \mu=\mu$ and $T^{* *} \mu_{0} \leqq \mu_{0}$. Thus $T^{* *}\left(\mu-\mu_{0}\right) \geqq \mu-\mu_{0}$. But, since $\left\|T^{* *}\right\|=\|T\| \leqq 1, T^{* *}\left(\mu-\mu_{0}\right)=$ $\mu-\mu_{0}$ and $T^{* *} \mu_{0}=\mu_{0}$. Therefore $g=d \mu_{0} / d m\left(\in L_{1}(X)\right)$ is $\Sigma$-fixed, and hence $g=0$ a.e. on $X$, by (0). It now follows from Lemma 1 of [18] that there exists a function $u \in L_{\infty}(X)$ satisfying

$$
u>0 \text { a.e. on } X \text { and }\langle\mu, u\rangle=\varphi\left(\left\langle T_{\alpha} 1, u\right\rangle\right)=0 \text {. }
$$

Thus we have $\inf _{T \in \Sigma} \int(T 1) u d m=0$.

(i) $\Rightarrow(0)$ : Let $g \in L_{1}(X)$ be such that $T g=g$ for all $T \in \Sigma$. Then, for each $T \in \Sigma, T|g| \geqq|g|$ and thus $T|g|=|g|$. By this and a standard approximation argument, we observe that (i) implies

$$
\int|g| u d m=\inf _{T \in \Sigma} \int(T|g|) u d m \leqq \inf _{T \in \Sigma} \int(T 1) u d m=0 .
$$

Hence $\int|g| u d m=0$, and thus $g=0$ a.e. on $X$, since $u>$ a.e. on $X$. 
The implication (i) $\Rightarrow$ (iii) is a direct consequence of Lemma 2, and the implications (iii) $\Rightarrow$ (ii) $\Rightarrow$ (i) and (iv) $\Rightarrow$ (i) are obvious. If $\left(T_{\alpha}, \alpha \in \Lambda\right)$ is a uniformly left $\Sigma$-ergodic net, then the implication (iii) $\Rightarrow$ (iv) follows from Lemma 3 .

The proof is complete.

3. Invariant measure for ergodic semigroups. In this section we investigate the invariant measure problem for weakly or uniformly ergodic semigroups $\Sigma=\{T\}$. For this purpose we need the following decomposition, which is a generalization of Sucheston's [26] obtained for power bounded positive linear operators on $L_{1}(X)$. See also Derriennic and Lin [5] and the author [22], [24], [25].

LEMMA 4. Let $\left(T_{\alpha}, \alpha \in \Lambda\right)$ be a weakly right $\Sigma$-ergodic net. Then the space $X$ decomposes into two measurable sets $Y$ and $Z$ such that

(i) if $f \in L_{1}(Z)$, then $T f \in L_{1}(Z)$ and $T_{\alpha} f \in L_{1}(Z)$ for all $T \in \Sigma$ and all $\alpha \in \Lambda$, and furthermore

$$
\inf _{T \in \Sigma}\|T f\|_{1}=0=\liminf _{\alpha}\left\|T_{\alpha} f\right\|_{1},
$$

(ii) there exists a nonnegative function $e \in L_{\infty}(X)$ with supp $e=$ $Y$ and $T^{*} e=e=T_{\alpha}^{*} e$ for all $T \in \Sigma$ and all $\alpha \in \Lambda$.

In particular, if $\left(T_{\alpha}, \alpha \in \Lambda\right)$ is a strongly right $\Sigma$-ergodic net, then $\lim _{\alpha}\left\|T_{\alpha} f\right\|_{1}=0$ for all $f \in L_{1}(Z)$.

Proof. It is easy to see that there exists a nonnegative function $e \in L_{\infty}(X)$, with $T^{*} e=e$ for all $T \in \Sigma$, such that $0 \leqq u \in L_{\infty}(X)$ and $T^{*} u=u$ for all $T \in \Sigma$ imply $\operatorname{supp} u \subset \operatorname{supp} e$. Let $Y=\operatorname{supp} e$ and $Z=X-Y$. Since for every $f \in L_{1}(X)$ and every $\alpha \in \Lambda, T_{\alpha} f \in \overline{\mathrm{co}} \Sigma f$, it follows that $T_{\alpha}^{*} e=e$ for all $\alpha \in \Lambda$. To prove (i), let $\varphi \in \mathrm{GL}(\Lambda)$ and choose a nonnegative function $u \in L_{\infty}(X)$ so that

$$
\langle f, u\rangle=\varphi\left(\left\langle T_{\alpha} f, 1\right\rangle\right) \text { for all } f \in L_{1}(X) \text {. }
$$

Since $\left(T_{\alpha}, \alpha \in \Lambda\right)$ is a weakly right $\Sigma$-ergodic net, it then follows that $T^{*} u=u$ for every $T \in \Sigma$. Therefore $\operatorname{supp} u \subset \operatorname{supp} e=Y$, and hence, for $0 \leqq f \in L_{1}(Z)$, we have

$$
\inf _{T \in \Sigma}\|T f\|_{1} \leqq \liminf _{\alpha}\left\|T_{\alpha} f\right\|_{1} \leqq\langle f, u\rangle=0,
$$

which proves the second part of (i). The first part of (i) is a direct consequence of (ii).

In particular, if $\left(T_{\alpha}, \alpha \in \Lambda\right)$ is a strongly right $\Sigma$-ergodic net, then, for each $T \in \Sigma$ and each $f \in L_{1}(Z)$, 


$$
\begin{aligned}
\limsup _{\alpha}\left\|T_{\alpha} f\right\|_{1} & \leqq \lim _{\alpha} \sup \left(\left\|T_{\alpha} T f-T_{\alpha} f\right\|_{1}+\left\|T_{\alpha} T f\right\|_{1}\right) \\
& \leqq\left(\lim \sup _{\alpha}\left\|T_{\alpha}\right\|\right)\|T f\|_{1} .
\end{aligned}
$$

Hence, by (i), we obtain

$$
\limsup _{\alpha}\left\|T_{\alpha} f\right\|_{1} \leqq\left(\lim _{\alpha} \sup \left\|T_{\alpha}\right\|\right)\left(\inf _{T \in \Sigma}\|T f\|_{1}\right)=0 .
$$

The proof is complete.

Let us now assume that $\left(T_{\alpha}, \alpha \in \Lambda\right)$ is a weakly $\Sigma$-ergodic net, and let $Y, Z$, and $e$ be as in Lemma 4. If $T \in \Sigma$ and $\alpha \in \Lambda$, define

$$
T^{\prime}(e f)=e(T f) \text { and } T_{\alpha}^{\prime}(e f)=e\left(T_{\alpha} f\right) \quad\left(f \in L_{1}(Y)\right) .
$$

Then, since $\left\{e f: f \in L_{1}(Y)\right\}$ is a dense subspace of $L_{1}(Y)$, and since $\left\|T^{\prime}(e f)\right\|_{1} \leqq\|e f\|_{1}$ and $\left\|T_{\alpha}^{\prime}(e f)\right\|_{1} \leqq\|e f\|_{1}$ for all $f \in L_{1}(Y), T^{\prime}$ and $T_{\alpha}^{\prime}$ can be regarded as positive linear contraction operators on $L_{1}(Y)$. Clearly, $\Sigma^{\prime}=\left\{T^{\prime}: T \in \Sigma\right\}$ is a semigroup. Furthermore, for all $f \in$ $L_{1}(Y)$ and all $\alpha \in \Lambda$,

$$
T_{\alpha}^{\prime}(e f) \in \overline{\mathrm{co}} \Sigma^{\prime}(e f)
$$

and

weak- $\lim _{\alpha}\left(T_{\alpha}^{\prime} T^{\prime}-T_{\alpha}^{\prime}\right)(e f)=0=$ weak- $\lim _{\alpha}\left(T^{\prime} T_{\alpha}^{\prime}-T_{\alpha}^{\prime}\right)(e f)$.

Thus, by an approximation argument, $\left(T_{\alpha}^{\prime}, \alpha \in \Lambda\right)$ is a weakly $\Sigma^{\prime}$ ergodic net. It is immediate that if $\left(T_{\alpha}, \alpha \in \Lambda\right)$ is strongly $\Sigma$-ergodic, then $\left(T_{\alpha}^{\prime}, \alpha \in \Lambda\right)$ is strongly $\Sigma^{\prime}$-ergodic.

THEOREM 4. Let $\left(T_{\alpha}, \alpha \in \Lambda\right)$ be a weakly $\Sigma$-ergodic net. Then the following conditions are equivalent:

(0) There exists a $\Sigma$-fixed $f_{0} \in L_{1}(X)$ with $f_{0}>0$ a.e. on $X$.

(i) $A \in \mathfrak{M}$ and $m(A)>0$ imply $\Delta\left(\int_{A} T_{\alpha} 1 d m\right)>0$.

Proof. By virtue of Theorem 1, it is enough to show that (i) implies (0).

Assume that (i) holds. It then follows from an approximation argument that

$$
\Delta\left(\int_{A} T_{\alpha}^{\prime} 1_{Y} d m\right)>0
$$

for any $A \in \mathfrak{M}$ with $A \subset Y$ and $m(A)>0$. By this and Theorem 2 and the fact that $e>0$ a.e. on $Y$, we observe that 


$$
\inf _{\alpha \in \Lambda} \int_{A} T_{\alpha} 1 d m \geqq \inf _{\alpha \in A} \int_{A} T_{\alpha} 1_{Y} d m=\inf _{\alpha \in A} \int_{A}\left(T_{\alpha}^{\prime} e\right) / e d m>0
$$

for any $A \in \mathfrak{M}$ with $A \subset Y$ and $m(A)>0$. (A similar but more complete vesrion of this argument appeared in the proof of Lemma 1 of Fong [9].) Hence the argument in the proof of (i) $\Rightarrow(0)$ in Theorem 1 can be applied to infer that there exists a nonnegative $\Sigma$-fixed $f_{0} \in L_{1}(X)$, with $f_{0}>0$ a.e. on $Y$.

Let $F=X-\operatorname{supp} f_{0}$ and let $\phi \in \mathrm{GL}(\Lambda)$. Since $T_{\alpha} f_{0}=f_{0}$ for every $\alpha \in \Lambda$, it follows that

$$
\varphi\left(\int_{F} T_{\alpha} 1 d m\right) \leqq \varphi\left(\int T_{\alpha} 1_{F} d m\right),
$$

and the weak $\Sigma$-ergodicity of the net $\left(T_{\alpha}, \alpha \in \Lambda\right)$ implies that

$$
\varphi\left(\int T_{\alpha} 1_{F} d m\right)=\varphi\left(\int T_{\alpha} T 1_{F} d m\right)
$$

for every $T \in \Sigma$. Hence $\varphi\left(\int_{F} T_{\alpha} 1 d m\right)=0$, since Lemma 4 asserts that inf $\left\{\left\|T 1_{F}\right\|_{1}: T \in \Sigma\right\}=0$, and since lim $\sup _{\alpha}\left\|T_{\alpha}\right\|<\infty$. Consequently we have $m(F)=0$, by (i).

This completes the proof.

LemMa 5. Suppose $\left(T_{\alpha}, \alpha \in \Lambda\right)$ is a uniformly $\Sigma$-ergodic net. If $0 \leqq u \in L_{\infty}(Y)$ satisfies $\sum_{n=1}^{\infty} T_{n}^{* *} u \in L_{\infty}(Y)$ for some sequence $\left(T_{n}^{\prime}, n \geqq 1\right)$ in $\Sigma^{\prime}=\left\{T^{\prime}: T \in \Sigma\right\}$, then $\lim \sup _{\alpha} T_{\alpha}^{*} u=0$ a.e. on $Y$.

Proof. Fix an $n \geqq 1$, and define

$$
E(n)=\{x \in Y: e(x) \geqq 1 / n\} .
$$

Given an $\varepsilon>0$, write, for each $\alpha \in \Lambda$,

$$
E_{\alpha}(n)=\left\{x \in E(n): T_{\alpha}^{*} u(x) \geqq \varepsilon\right\} \text {. }
$$

Then, as in the proof of Lemma 3, we see that there exists an $\alpha_{0} \in \Lambda$ such that if $\alpha \geqq \alpha_{0}$ then $m\left(E_{\alpha}(n)\right)=0$. Hence it follows that

$$
\lim _{\alpha}\left\|\left(T_{\alpha}^{\prime *} u\right) 1_{E(n)}\right\|_{\infty}=0 .
$$

This completes the proof, since $Y=\bigcup_{n=1}^{\infty} E(n)$.

LEMMA 6. Let $\left(T_{\alpha}, \alpha \in \Lambda\right)$ be a weakly right $\Sigma$-ergodic net, and let $Y, Z$, and $e$ be as in Lemma 4. Suppose $\left(S_{r}, \gamma \in \Gamma\right)$ is a net of positive linear operators on $L_{1}(X)$ satisfying

(a) $\lim \sup \left\|S_{r}\right\|<\infty$,

(b) for every $f \in L_{1}(X)$ and every $\gamma \in \Gamma, S_{\gamma} f \in \overline{c o} \Sigma f$,

(c) for every $T \in \Sigma$, there corresponds a net $\left(R_{r}, \gamma \in \Gamma\right)$ of positive 
linear operators on $L_{1}(X)$ with

$$
\lim _{\gamma}\left\|R_{\gamma}\right\|=0 \text { and } S_{\gamma} T-S_{\gamma}+R_{\gamma} \geqq 0 \text { for all } \gamma \in \Gamma .
$$

Define $s=\lim \sup _{r} S_{r}^{*} 1$. Then $0 \leqq s \in L_{\infty}(X)$, supp $s=Y, T^{*} s \geqq s$ for all $T \in \Sigma$, and $T_{\alpha}^{*} s \geqq s$ for all $\alpha \in \Lambda$.

Proof. It is clear that $0 \leqq s \in L_{\infty}(X)$. If $T \in \Sigma$, then we have

$$
\begin{aligned}
T^{*} s & =T^{*}\left(\lim _{r} \sup S_{r}^{* 1} 1\right) \geqq \lim _{r} \sup \left(S_{r} T\right)^{* 1} \\
& \geqq \lim _{r} \sup \left(S_{r}^{*} 1-R_{r}^{* 1} 1\right)=\lim _{r} \sup S_{r}^{*} 1=s,
\end{aligned}
$$

from which we also have $T_{\alpha}^{*} s \geqq s$ for all $\alpha \in \Lambda$, since $T_{\alpha} f \in \overline{\mathrm{co}} \Sigma f$ for all $f \in L_{1}(X)$ and all $\alpha \in \Lambda$. Now, let $0 \leqq f \in L_{1}(Z)$. Then, since $\langle f, s\rangle \leqq\left\langle f, T^{*} s\right\rangle \leqq\|T f\|_{1}\|s\|_{\infty}$ for all $T \in \Sigma$, and since

$$
\inf \left\{\|T f\|_{1}: T \in \Sigma\right\}=0
$$

by Lemma $4,\langle f, s\rangle=0$. This proves $s=0$ a.e. on $Z$. On the other hand, (b) implies $S_{r}^{*} e=e$ for all $\gamma \in \Gamma$, hence we have supp $e \subset \operatorname{supp} s$.

This completes the proof.

Theorem 5. Suppose $\left(T_{\alpha}, \alpha \in \Lambda\right)$ is a uniformly $\Sigma$-ergodic net. Then the following conditions are equivalent:

(0) There exists a $\Sigma$-fixed $f_{0} \in L_{1}(X)$ with $f_{0}>0$ a.e. on $X$.

(i) $A \in \mathfrak{M}$ and $m(A)>0$ imply $\lim \sup _{\alpha} \int_{A} T_{\alpha} 1 d \dot{m}>0$.

(ii) $A \in \mathfrak{M}$ and $m(A)>0$ imply $\lim \sup _{\alpha} T_{\alpha}^{*} 1_{A} \not \equiv 0$.

Proof. $(0) \Rightarrow$ (i): Immediate from Theorem 1 .

(i) $\Rightarrow$ (ii): Let $A \in \mathbb{M}$ satisfy $\lim \sup _{\alpha} T_{\alpha}^{*} 1_{A}=0$ a.e. on $X$. Then we can choose a sequence $\left(\alpha_{n}, n \geqq 1\right)$ in $\Lambda$, with $\alpha_{n+1} \geqq \alpha_{n}$ for every $n$, such that

$$
\lim _{n \rightarrow \infty}\left(\underset{\alpha \geq \alpha_{n}}{\operatorname{ess} \sup _{\alpha}} T_{\alpha}^{*} 1_{A}\right)=0 \text { a.e. on } X \text {. }
$$

Then, by Fatou's lemma and by Lebesgue's bounded convergence theorem,

$$
\lim \sup _{\alpha} \int_{A} T_{\alpha} 1 d m \leqq \lim _{n \rightarrow \infty} \int\left(\underset{\alpha \geqq \alpha_{n}}{\operatorname{ess} \sup _{\alpha}} T_{\alpha}^{*} 1_{A}\right) d m=0 .
$$

Hence (i) implies $m(A)=0$.

(ii) $\Rightarrow(0)$ : Let $0 \leqq u \in L_{\infty}(Y)$ satisfy $\sum_{n=1}^{\infty} T_{n}^{*} u \in L_{\infty}(Y)$ for some sequence $\left(T_{n}^{\prime}, n \geqq 1\right)$ in $\Sigma^{\prime}=\left\{T^{\prime}: T \in \Sigma\right\}$. Then, by Lemma 5 , $\lim \sup _{\alpha} T_{\alpha}^{\prime *} u=0$ a.e. on $Y$. Since $T_{\alpha}^{\prime *} u=(1 / e) T_{\alpha}^{*}(e u)$ for all $\alpha \in \Lambda$, we 
then observe that $\lim \sup _{\alpha} T_{\alpha}^{*}(e u)=0$ a.e. on $X$. Since $e>0$ a.e. on $Y$, this and (ii) imply $u=0$ a.e. on $X$. Therefore, using Lemma 2, we see that

$$
\inf _{T \in \Sigma} \int_{A} T 1 d m>0
$$

for any $A \in \mathfrak{M}$ with $A \subset Y$ and $m(A)>0$ (cf. the proof of (i) $\Rightarrow(0)$ in Theorem 4). Hence, as in the proof of (i) $\Rightarrow(0)$ in Theorem 1 , we can find a nonnegative $\Sigma$-fixed $f_{0} \in L_{1}(X)$, with $f_{0}>0$ a.e. on $Y$. Let $F=X-\operatorname{supp} f_{0}$. To prove that $m(F)=0$, define

$$
s=\lim _{\alpha} \sup T_{\alpha}^{*} 1 \text {. }
$$

Since $\lim _{\alpha}\left\|T_{\alpha} T-T_{\alpha}\right\|=0$ for every $T \in \Sigma$, Lemma 6 can be applied to $\left(T_{\alpha}, \alpha \in \Lambda\right)$ instead of $\left(S_{\gamma}, \gamma \in \Gamma\right)$ to infer that supp $s=Y$ (cf. Chacon and Krengel [1]). It follows that $\lim \sup _{\alpha} T_{\alpha}^{*} 1_{F}=0$ a.e. on $X$, since $T_{\alpha}^{*} 1_{F}=0$ a.e. on $\operatorname{supp} f_{0}$ and $s=0$ a.e. on $F=X-\operatorname{supp} f_{0} \subset X-Y$. Hence (ii) implies $m(F)=0$.

This completes the proof.

4. Examples. In this section we apply the above general results to explain some special examples. The first two theorems are concerned with the invariant measure problem for a single operator $T$ and the last two theorems are concerned with the problem for a oneparameter semigroup $\Sigma=\left\{T_{t}: 0<t<\infty\right\}$.

THEOREM 6. Suppose $T$ is a positive linear operator on $L_{1}(X)$ such that

$$
\sup _{0<\lambda<1}\left\|(1-\lambda) \sum_{n=0}^{\infty} \lambda^{n} T^{n}\right\|<\infty \text {. }
$$

Then the following conditions are equivalent: $X$.

(0) There exists an $f_{0} \in L_{1}(X)$ with $T f_{0}=f_{0}$ and $f_{0}>0$ a.e. on

(i ) $A \in \mathfrak{M}$ and $m(A)>0$ imply

$$
\liminf _{\lambda \rightarrow 1-0}(1-\lambda) \times \sum_{n=0}^{\infty} \lambda^{n} T^{* n} 1_{A} \not \equiv 0 \text {. }
$$

(ii) $A \in \mathfrak{M}$ and $m(A)>0$ imply lim $\sup _{\lambda \rightarrow 1-0}(1-\lambda) \times \sum_{n=0}^{\infty} \lambda^{n} T^{* n} 1_{A} \not \equiv 0$. In particular, if $\|T\| \leqq 1$, then the following condition and the above three conditions are still equivalent:

(iii) $A \in \mathfrak{M}$ and $m(A)>0$ imply

$$
\limsup _{\lambda \rightarrow 1-0}\left\|(1-\lambda) \times \sum_{n=0}^{\infty} \lambda^{n} T^{* n} 1_{A}\right\|_{\infty}>0 \text {. }
$$


Proof. Write $T_{\lambda}=(1-\lambda) \sum_{n=0}^{\infty} \lambda^{n} T^{n}$ for $0<\lambda<1$. Then, by (3), we have

$$
\lim _{\lambda \rightarrow 1-0}\left\|T T_{\lambda}-T_{\lambda}\right\|=0 \text {. }
$$

Thus $\left(T_{\lambda}, 0<\lambda<1\right)$ is a uniformly $\Sigma$-ergodic net, with $\Sigma=\left\{T^{n}: n \geqq 0\right\}$, and let $Y, Z$, and $e$ be as in Lemma 4. Then the proof proceeds as follows.

$(0) \Rightarrow$ (i): By the Radon-Nikodym theorem, we may and do assume without loss of generality that $T 1=1$. Then, since

$$
\left\|T^{*} f\right\|_{1} \leqq \int T^{*}|f| d m=\int|f| d m \text { for all } f \in L_{\infty}(X),
$$

$T^{*}$ can be extended to a positive linear contraction operator $S$ on $L_{1}(X)$. It is clear that $S^{*} f=T f$ for all $f \in L_{\infty}(X)$. Since $S e=T^{*} e=e$, supp $e=Y$, and

$$
\inf _{n \geq 0}\left\|S^{* n} 1_{Z}\right\|_{1}=\inf _{n \geq 0}\left\|T^{n} 1_{Z}\right\|_{1}=0
$$

by Lemma 4 , it follows from standard arguments that $Y$ and $Z$ are the conservative and dissipative parts (cf. [8]) of $S$, respectively. Hence, using Chacon-Ornstein's ratio ergodic theorem [2], we see that for any $f \in L_{\infty}(X)$ the limit

$$
\lim _{n \rightarrow \infty} \frac{1}{n} \sum_{i=1}^{n-1} T^{* i} f=\lim _{n \rightarrow \infty} \frac{1}{n} \sum_{i=0}^{n-1} S^{i} f
$$

exists a.e. on $X$.

Let $A \in \mathfrak{M}$ with $m(A)>0$ be given. Then

$$
m(A)=\int_{Y} T^{* n} 1_{A} d m+\int_{A} T^{n} 1_{Z} d m \text { for all } n \geqq 0,
$$

and by Lemma 4,

$$
\inf _{n \geqq 0} \int_{A} T^{n} 1_{Z} d m \leqq \inf _{n \geqq 0}\left\|T^{n} 1_{Z}\right\|_{1}=0 .
$$

Thus $T^{* k} 1_{A} \geqq f$ for some $k \geqq 0$ and some $0 \leqq f \in L_{\infty}(X)$, with supp $f \subset$ $Y$ and $\|f\|_{1}>0$. Therefore

$$
\lim _{n \rightarrow \infty} \frac{1}{n} \sum_{i=0}^{n-1} T^{* i} 1_{A} \geqq \lim _{n \rightarrow \infty} \frac{1}{n} \sum_{i=0}^{n-1} T^{* i} f \text { a.e. on } X,
$$

and

$$
\lim _{n \rightarrow \infty} \frac{1}{n} \sum_{i=0}^{n-1} T^{* i} f \not \equiv 0,
$$

since $\left\langle 1,1 / n \sum_{i=0}^{n-1} T^{* \imath} f\right\rangle=\langle 1, f\rangle \neq 0$ for all $n \geqq 0$. Hence 


$$
\lim _{\lambda \rightarrow 1-0}(1-\lambda) \sum_{n=0}^{\infty} \lambda^{n} T^{* n} 1_{A}=\lim _{n \rightarrow \infty} \frac{1}{n} \sum_{i=0}^{n-1} T^{* i} 1_{A} \not \equiv 0
$$

by a well-known theorem of the theory of summability (cf. Zygmund [28], Theorem (1.33), p. 80), and thus we have proved that (0) implies (i).

The implications (i) $\Rightarrow$ (ii) $\Rightarrow$ (iii) are immediate. The implication (ii) $\Rightarrow(0)$ follows from Theorem 5 , and if $\|T\| \leqq 1$, then the implication (iii) $\Rightarrow(0)$ follows from Theorems 1 and 2 .

The proof is complete.

For a positive linear operator $T$ on $L_{1}(X)$ and a real number $r>-1$, we denote by $\sigma_{n}^{r}(T), n=0,1, \cdots$, the Cesàro means of order $r$ of the sequence $\left(T^{n}, n \geqq 0\right)$, i.e.,

$$
\sigma_{n}^{r}(T)=\frac{1}{\left(\begin{array}{c}
r+n \\
n
\end{array}\right)} \sum_{i=0}^{n}\left(\begin{array}{c}
r+i-1 \\
i
\end{array}\right) T^{n-i} \quad(n \geqq 0) .
$$

The next theorem is a generalization of results due to Foguel [8] and Derriennic and Lin [5]; Foguel considered the case $\|T\| \leqq 1$, and Derriennic and Lin considered the case $\sup _{n \geqq 0}\left\|\sigma_{n}^{1}(T)\right\|<\infty$.

THEOREM 7. Let $T$ be a positive linear operator on $L_{1}(X)$ and $r$ a positive real number. If

$$
\sup _{n \geq 0}\left\|\sigma_{n}^{r}(T)\right\|<\infty,
$$

then the following conditions are equivalent: $X$.

(0) There exists an $f_{0} \in L_{1}(X)$ with $T f_{0}=f_{0}$ and $f_{0}>0$ a.e. on

(i ) $A \in \mathfrak{M}$ and $m(A)>0$ imply lim $\sup _{n \rightarrow \infty} \sigma_{n}^{r}(T)^{*} 1_{A} \not \equiv 0$.

In particular, if $\|T\| \leqq 1$, then the following condition and the above two conditions are still equivalent:

(ii) $A \in \mathfrak{M}$ and $m(A)>0$ imply $\lim \sup _{n \rightarrow \infty}\left\|\sigma_{n}^{r}(T)^{*} 1_{A}\right\|_{\infty}>0$.

Proof. An elementary computation (cf. [28], Chapter III) shows that, for any $\lambda>0$,

$$
T \sigma_{n}^{\lambda}(T)-\sigma_{n}^{\lambda}(T)=\frac{\lambda}{n+1}\left[\sigma_{n+1}^{\lambda-1}(T)-I\right] \quad(n \geqq 0) .
$$

Applying this equation to $\lambda=r+1$, we observe that $\left(\sigma_{n}^{r+1}(T), n \geqq 0\right)$ is a uniformly $\Sigma$-ergodic sequence, with $\Sigma=\left\{T^{n}: n \geqq 0\right\}$. (This argument is due to Lloyd. See [15].) Let $Y, Z$, and $e$ be as in Lemma 4. Then the proof proceeds as follows.

(i) $\Rightarrow(0)$ : Let $T^{\prime}$ be the positive linear contraction operator on 
$L_{1}(Y)$ satisfying

$$
T^{\prime}(e f)=e(T f) \text { for all } f \in L_{1}(Y) .
$$

Then we see that

$$
\lim _{n \rightarrow \infty}\left\|T^{\prime} \sigma_{n}^{r}\left(T^{\prime}\right)-\sigma_{n}^{r}\left(T^{\prime}\right)\right\|=\lim _{n \rightarrow \infty} \frac{r}{n+1}\left\|\sigma_{n+1}^{r-1}\left(T^{\prime}\right)-I\right\|=0 .
$$

In fact, if $r \geqq 1$, then this is immediate. If $0<r<1$, then, writing $A_{n}^{\lambda}=\left(\begin{array}{c}\lambda+n \\ n\end{array}\right)$ for $\lambda>-1$ and $n \geqq 0$, we have

$$
\begin{gathered}
T^{\prime} \sigma_{n}^{r}\left(T^{\prime}\right)-\sigma_{n}^{r}\left(T^{\prime}\right)=\frac{r}{n+1}\left[\frac{1}{A_{n+1}^{r-1}} \sum_{i=0}^{n+1} A_{i}^{r-2} T^{\prime n-\imath}-I\right], \\
\sum_{n=0}^{\infty}\left|A_{n}^{r-2}\right|<\infty, \text { and } \lim _{n \rightarrow \infty} n A_{n}^{r-1}=\infty ;
\end{gathered}
$$

therefore we obtain the desired conclusion. Hence $\left(\sigma_{n}^{r}\left(T^{\prime}\right), n \geqq 0\right)$ is a uniformly $\Sigma^{\prime}$-ergodic sequence, with $\Sigma^{\prime}=\left\{T^{\prime n}: n \geqq 0\right\}$. Since

$$
\sigma_{n}^{r}\left(T^{\prime}\right)^{*} f=(1 / e) \sigma_{n}^{r}(T)^{*}(e f) \text { for all } f \in L_{\infty}(Y),
$$

(i) implies that if $A \in \mathfrak{M}, A \subset Y$ and $m(A)>0$, then

$$
\limsup _{n \rightarrow \infty} \sigma_{n}^{r}\left(T^{\prime}\right)^{*} 1_{A} \not \equiv 0 \text {. }
$$

Therefore, applying Theorem 2 to $\Sigma^{\prime}$ and to $\left(\sigma_{n}^{r}\left(T^{\prime}\right), n \geqq 0\right)$, and doing as in the proof of (ii) $\Rightarrow(0)$ in Theorem 5 , we observe that (i) implies the existence of a nonnegative $f_{0} \in L_{1}(X)$, with $T f_{0}=f_{0}$ and $f_{0}>0$ a.e. on $Y$.

Let $F=X-\operatorname{supp} f_{0}$ and $s=\lim \sup _{n \rightarrow \infty} \sigma_{n}^{r}(T)^{*} 1$. An elementary computation shows that there exists a sequence $\left(R_{n}, n \geqq 0\right)$ of positive linear operators on $L_{1}(X)$ such that

$$
T \sigma_{n}^{r}(T)-\sigma_{n+1}^{r}(T)+R_{n} \geqq 0 \text { for all } n \geqq 0,
$$

and

$$
\lim _{n \rightarrow \infty}\left\|R_{n}\right\|=0
$$

Hence it follows from a slight modification of the proof of Lemma 6 that $\operatorname{supp} s=Y$, and therefore

$$
\limsup _{n \rightarrow \infty} \sigma_{n}^{r}(T)^{*} 1_{F}=0 \text { a.e. on } X \text {, }
$$

since $\sigma_{n}^{r}(T)^{*} 1_{F}=0$ a.e. on $\operatorname{supp} f_{0}$ and $s=0$ a.e. on $F=X-\operatorname{supp} f_{0}: \subset$ $X-Y$. This and (i) imply $m(F)=0$, and hence we have proved that (i) implies (0). 
The implications $(0) \Rightarrow$ (i) $\Rightarrow$ (ii) are immediate from Fatou's lemma. If $\|T\| \leqq 1$, then the implication (ii) $\Rightarrow(0)$ follows from Theorem 2 , since $\left(\sigma_{n}^{r}(T), n \geqq 0\right)$ is a uniformly $\Sigma$-ergodic sequence, with $\Sigma=$ $\left\{T^{n}: n \geqq 0\right\}$.

The proof is complete.

A one-parameter semigroup $\Sigma=\left\{T_{t}: 0<t<\infty\right\}$ (i.e., $T_{t} T_{t^{\prime}}=T_{t+t^{\prime}}$ for all $\left.t, t^{\prime}>0\right)$ of positive linear operators on $L_{1}(X)$ is said to be strongly continuous if, for every $f \in L_{1}(X)$ and every $t^{\prime}>0$,

$$
\lim _{t \rightarrow t^{\prime}}\left\|T_{t} f-T_{t^{\prime}} f\right\|_{1}=0 \text {. }
$$

In the remainder of the paper weshall assume that $\Sigma=\left\{T_{t}: 0<t<\infty\right\}$ is strongly continuous. Then, by an approximation argument (cf. Ornstein [19], §4), we observe that, given an $f \in L_{1}(X)$, there exists a scalar function $g(t, x)$ defined on $(0, \infty) \times X$, measurable with respect to the product of Lebesgue measure and $m$, such that for each $t>0, g(t, x)$, as a function of $x$, belongs to the equivalence class of $T_{t} f$. In the sequel, $g(t, x)$ will be denoted by $T_{t} f(x)$. Using Fubini's theorem, we then see that there exists a measurable set $E(f) \subset X$ with $m(E(f))=0$, dependent on $f$ but independent of $t$, such that if $x \notin E(f)$ then the scalar function $t \mapsto T_{t} f(x)$ is Lebesgue integrable on every finite interval $(a, b)$, with $0<a<b<\infty$, and the integral $\int_{a}^{b} T_{t} f(x) d t$, as a function of $x$, belongs to the equivalence class of $\int_{a}^{b} T_{t} f d t\left(\in L_{1}(X)\right)$, where $\int_{a}^{b} T_{t} f d t$ denotes the Bochner integral of the vector valued function $t \mapsto T_{t} f$ with respect to Lebesgue measure on the interval $(a, b)$.

Similarly, if there exist nonnegative real numbers $\lambda_{0}$ and $\alpha$ such that, for all $\lambda>\lambda_{0}$, the vector valued function $t \mapsto e^{-\lambda t} T_{t} f$ is Bochner integrable on the interval $(\alpha, \infty)$, then there exists a measurable set $E^{\prime}(f) \subset X$ with $m\left(E^{\prime}(f)\right)=0$, dependent on $f$ but independent of $t$, such that if $x \notin E^{\prime}(f)$ then, for all $\lambda>\lambda_{0}$, the scalar function $t \mapsto$ $e^{-\lambda t} T_{t} f(x)$ is Lebesgue integrable on the interval $(a, \infty)$, and the integral $\int_{a}^{\infty} e^{-\lambda t} T_{t} f(x) d t$, as a function of $x$, belongs to the equivalence class of $\int_{a}^{\infty} e^{-\lambda t} T_{t} f d t\left(\in L_{1}(X)\right)$.

Next, let $\Sigma^{*}=\left\{T_{t}^{*}: 0<t<\infty\right\}$ denote the adjoint semigroup of $\Sigma$. Therefore $\Sigma^{*}$ acts on $L_{\infty}(X)$, and $\left\langle u, T_{t}^{*} f\right\rangle=\left\langle T_{t} u, f\right\rangle$ for all $u \epsilon$ $L_{1}(X)$, all $f \in L_{\infty}(X)$, and all $t>0$. For $0<a<b<\infty$, the integral $\int_{a}^{b} T_{t}^{*} f d t\left(\in L_{\infty}(X)\right)$ for $f \in L_{\infty}(X)$ is defined by the relation:

$$
\left\langle u, \int_{a}^{b} T_{t}^{*} f d t\right\rangle=\left\langle\int_{a}^{b} T_{t} u d t, f\right\rangle \quad\left(u \in L_{1}(X)\right) .
$$


Similarly, if $\lambda>0$ and if, for all $u \in L_{1}(X)$, the vector valued function $t \mapsto e^{-\lambda t} T_{t} u$ is Bochner integrable on some interval $(\alpha, \infty)$, then the mapping $u \mapsto \int_{a}^{\infty} e^{-\lambda t} T_{t} u d t$ is a positive linear operator on $L_{1}(X)$, and hence the integral $\int_{a}^{\infty} e^{-\lambda . t} T_{t}^{*} f d t\left(\in L_{\infty}(X)\right)$ for $f \in L_{\infty}(X)$ is still defined by the relation:

$$
\left\langle u, \int_{a}^{\infty} e^{-\lambda t} T_{t}^{*} f d t\right\rangle=\left\langle\int_{a}^{\infty} e^{-\lambda} T_{t} u d t, f\right\rangle \quad\left(u \in L_{1}(X)\right) .
$$

A slight modification of the proof of Theorem 1.1 of Lin [14] shows that, given an $f \in L_{\infty}(X)$, there exists a scalar function $g(t, x)$ defined on $(0, \infty) \times X$, measurable with respect to the product of Lebesgue measure and $m$, and a measurable subset $E(f) \subset X$ with $m(E(f))=0$, dependent on $f$ but independent of $t$, such that if $x \notin E(f)$ then the scalar function $t \mapsto g(t, x)$ is Lebesgue integrable on every finite interval $(a, b)$, with $0<a<b<\infty$, and the integral $\int_{a}^{b} g(t, x) d t$, as a function of $x$, belongs to the equivalence class of $\int_{a}^{b} T_{t}^{*} f d t$.

Similarly, if there exist nonnegative real numbers $\lambda_{0}$ and $a$ such that, for all $\lambda>\lambda_{0}$ and all $u \in L_{1}(X)$, the vector valued function $t \mapsto$ $e^{-\lambda t} T_{t} u$ is Bochner integrable on the interval $(\alpha, \infty)$, then there exists a measurable set $E^{\prime}(f) \subset X$ with $m\left(E^{\prime}(f)\right)=0$, dependent on $f$ but independent of $t$, such that if $x \notin E(f)$ then, for all $\lambda>\lambda_{0}$, the scalar function $t \mapsto e^{-\lambda t} g(t, x)$ is Lebesgue integrable on the interval $(\alpha, \infty)$, and the integral $\int_{\alpha}^{\infty} e^{-\lambda t} g(t, x) d t$, as a function of $x$, belongs to the equivalence class of $\int_{a}^{\infty} e^{-\lambda t} T_{t}^{*} f d t$.

In the sequel, $\int_{a}^{b} g(t, x) d t$ and $\int_{a}^{\infty} e^{-\lambda t} g(t, x) d t$ will be denoted by $\int_{a}^{b} T_{t}^{*} f(x) d t$ and $\int_{a}^{\infty} e^{-\lambda t} T_{t}^{*} f(x) d t$, respectively.

The next theorem is a generalization of results due to Lin [14] and the author [24].

Theorem 8. Let $\Sigma=\left\{T_{t}: 0<t<\infty\right\}$ be a strongly continuous one-parameter semigroup of positive linear operators on $L_{1}(X)$ and $r$ a positive real number. If

$$
\sup _{b>r}\left\|\frac{1}{b} \int_{r}^{b} T_{t} f d t\right\|_{1}<\infty \quad \text { for all } f \in L_{1}(X),
$$

then the following conditions are equivalent:

(0) There exists a $\Sigma$-fixed $f_{0} \in L_{1}(X)$ with $f_{0}>0$ a.e. on $X$.

(i ) $A \in \mathfrak{M}$ and $m(A)>0$ imply $\lim \inf _{b \rightarrow \infty} 1 / b \int_{r}^{b} T_{r}^{*} 1_{A}(x) d t \not \equiv 0$.

(ii) $A \in \mathfrak{M}$ and $m(A)>0$ imply lim $\sup _{b \rightarrow \infty} 1 / b \int_{r}^{b} T_{t}^{*} 1_{A}(x) d t \not \equiv 0$. 
In particular, if $\left\|T_{t}\right\| \leqq 1$ for all $t>0$, then the following condition and the above three conditions are still equivalent:

(iii) $A \in \mathfrak{M}$ and $m(A)>0$ imply lim $\sup _{b \rightarrow \infty}\left\|1 / b \int_{r}^{b} T_{t}^{*} 1_{A}(x) d t\right\|_{\infty}>0$.

Proof. By the uniform boundedness principle (cf. [6], Corollary II.3.21, p. 66), we have a constant $K$ such that, for all $f \in L_{1}(X)$ and all $b>r$,

$$
\left\|\frac{1}{b} \int_{r}^{b} T_{t} f d t\right\|_{1} \leqq K\|f\|_{1}
$$

Define

$$
s(x)=\limsup _{b \rightarrow \infty} \frac{1}{b} \int_{r}^{b} T_{t}^{*} 1(x) d t \quad(x \in X) .
$$

It follows that $0 \leqq s \in L_{\infty}(X)$ and, by an easy computation, that $T_{t}^{*} s \geqq s$ for all $t>0$. Thus we can define another nonnegative function $e$ in $L_{\infty}(X)$ by the relation:

$$
e(x)=\lim _{b \rightarrow \infty} \frac{1}{b} \int_{r}^{b} T_{t}^{*} s(x) d t \quad(x \in X) .
$$

It is then clear that $T_{t}^{*} e=e$ for all $t>0$, and if we let $Y=$ supp $e(=\operatorname{supp} s)$ and $Z=X-Y$, then for any $f \in L_{1}(Z)$,

$$
T_{t} f \in L_{1}(Z) \text { for all } t>0 \text { and } \lim _{b \rightarrow \infty} \frac{1}{b} \int_{r}^{b}\left\|T_{t} f\right\|_{1} d t=0 .
$$

(Cf. [24].) Using this fact and the continuous version of ChaconOrnstein's ratio ergodic theorem (see, for example, Fong and Sucheston [10]), the implication (0) $\Rightarrow$ (i) follows as in the proof of $(0) \Rightarrow$ (i) in Theorem 6. The implications (i) $\Rightarrow$ (ii) $\Rightarrow$ (iii) are obvious, and the implication (ii) $\Rightarrow(0)$ follows as in the proof of $(\mathrm{i}) \Rightarrow(0)$ in Theorem 7. If $\left\|T_{t}\right\| \leqq 1$ for all $t>0$, then the implication (iii) $\Rightarrow(0)$ follows from Theorems 1 and 2 , because $\left(1 /(b-r) \int_{r}^{b} T_{t} d t, b>r\right)$ is a uniformly $\Sigma$-ergodic net.

The proof is complete.

THEOREM 9. Let $\Sigma=\left\{T_{t}: 0<t<\infty\right\}$ be a strongly continuous one-parameter semigroup of positive linear operators on $L_{1}(X)$ and $r$ a positive real number. If

$$
\sup _{\lambda>0}\left\|\lambda \int_{r}^{\infty} e^{-\lambda t} T_{t} f d t\right\|_{1}<\infty \text { for all } f \in L_{1}(X),
$$

then the following conditions are equivalent: 
(0) There exists a $\Sigma$-fixed $f_{0} \in L_{1}(X)$ with $f_{0}>0$ a.e. on $X$.

(i ) $A \in \mathfrak{M}$ and $m(A)>0$ imply $\lim \inf _{\lambda \rightarrow+0} \lambda \int_{r}^{\infty} e^{-\lambda t} T_{t}^{*} 1_{A}(x) d t \not \equiv 0$.

(ii) $A \in \mathfrak{M}$ and $m(A)>0$ imply lim $\sup _{\lambda \rightarrow+0} \lambda \int_{r}^{\infty} e^{-\lambda t} T_{t}^{*} 1_{A}(x) d t \not \equiv 0$.

In particular, if $\left\|T_{t}\right\| \leqq 1$ for all $t>0$, then the following condition and the above three conditions are still equivalent:

(iii) $A \in \mathfrak{M}$ and $m(A)>0$ imply

$$
\limsup _{\lambda \rightarrow+0}\left\|\lambda \int_{r}^{\infty} e^{-\lambda t} T_{t}^{*} 1_{A}(x) d t\right\|_{\infty}>0 \text {. }
$$

Proof. Write $R_{\lambda} f=\lambda \int_{r}^{\infty} e^{-\lambda t} T_{t} f d t$ for all $\lambda>0$ and all $f \in L_{1}(X)$. Then $R_{\lambda}$ is a positive linear operator on $L_{1}(X)$ and $\sup _{\lambda>0}\left\|R_{\lambda}\right\|<\infty$. Furthemore,

$$
\lim _{\lambda \rightarrow+0}\left\|T_{t} R_{\lambda}-R_{\lambda}\right\|=0 \text { for every } t>0 .
$$

Hence the remainder of the proof is now immediate from the above arguments, and we omit the details.

REMARK. It is easily seen that if $r$ is a positive real number, then (4) implies (3) and (5) implies (6).

\section{REFERENCES}

1. R. V. Chacon and U. Krengel, Linear modulus of a linear operator, Proc. Amer. Math. Soc., 15 (1964), 553-559.

2. R. V. Chacon and D. S. Ornstein, A general ergodic theorem, Illinois J. Math., 4 (1960), 153-160.

3. M. M. Day, Semigroups and amenability, in: Semigroups, (Proc. Sympos., Wayne State Univ., Detroit, Mich., 1968), pp. 5-53, Academic Press, Inc., New York, 1969.

4. D. W. Dean and L. Sucheston, On invariant measures for operators, Z. Wahrscheinlichkeitstheorie und Verw. Gebiete, 6 (1966), 1-9.

5. Y. Derriennic and M. Lin, On invariant measures and ergodic theorems for positive operators, J. Functional Analysis, 13 (1973), 252-267.

6. N. Dunford and J. T. Schwartz, Linear Operators, Part I: General Theory, Interscience Publishers, Inc., New York, 1958.

7. W. F. Eberlein, Abstract ergodic theorems and weak almost periodic functions, Trans. Amer. Math. Soc., 67 (1949), 217-240.

8. S. R. Foguel, The Ergodic Theory of Markov Processes, Van Nostrand Reihold Co., New York, 1969.

9. H. Fong, On invariant functions for positive operators, Colloq. Math., 22 (1970), 75-84.

10. H. Fong and L. Sucheston, On the ratio ergodic theorem for semigroups, Pacific J. Math., 39 (1971), 659-667.

11. E. Granirer, On finite equivalent invariant measures for semigroups of transformations, Duke Math. J., 38 (1971), 395-408.

12. A. Hajian and $\mathrm{Y}$. Ito, Weakly wandering sets and invariant measures for a group of transformations, J. Math. Mech., 18 (1968/69), 1203-1216. 
13. U. Krengel, Classification of states for operators, in: Proc. Fifth Berkeley Sympos. Math. Statist. and Probability (Berkeley, Calif., 1965/66), Vol. II, Part 2, pp. 415-429, University of California Press, Berkeley, 1967.

14. M. Lin, Semi-groups of Markov operators, Boll. Un. Mat. Ital., (4) 6 (1972), 20-44. 15. S. P. Lloyd, On the mean ergodic theorem of Sine, Proc. Amer. Math. Soc., 56 (1976), 121-126.

16. J. Neveu, Mathematical Foundations of the Calculus of Probability, Holden-Day, Inc., San Francisco, 1965.

17. - Sur l'existence de measures invariantes en théorie ergodique, C. R. Acad. Sci. Paris, 260 (1965), 393-396.

18. - Existence of bounded invariant measures in ergodic theory, in: Proc. Fifth Berkeley Sympos. Math. Statist. and Probability (Berkeley, Calif., 1965/66), Vol. II, Part 2, pp. 461-472, University of California Press, Berkeley, 1967.

19. D. S. Ornstein, The sums of iterates of a positive operator, in: Advances in Probability and Related Topics (Edited by P. Ney), Vol. 2, pp. 85-115, Marcel Dekker, Inc., New York, 1970.

20. W. Rudin, Functional Analysis, McGraw-Hill Book Co., New York, 1973.

21. U. Sachdeva, On finite invariant measure for semigroups of operators, Canad. Math. Bull., 14 (1971), 197-206.

22. R. Sato, Ergodic properties of bounded $L_{1}$-operators, Proc. Amer. Math. Soc., 39 (1973), 540-546.

23. - On the existence of positive invariant functions for semigroups of operators, Tôhoku Math. J., (2) 27 (1975), 187-196.

24. - Invariant measures for semigroups, Studia Math., 53 (1975), 129-134.

25. - Invariat measures for bounded amenable semigroups of operators, Proc. Japan Acad,, 52 (1976), 215-218.

26. L. Sucheston, On the ergodic theorem for positive operators I, Z. Wahrscheinlichkeitstheorie und Verw. Gebite, 8 (1966), 1-11.

27. W. Takahashi, Invariant functions for amenable semigroups of positive contractions on $L^{1}$, Kōdai Math. Sem. Rep., 23 (1971), 131-143.

28. A. Zygmund, Trigonometric Series, Vols. I and II, Cambridge University Press, Cambridge, 1968.

Received December 28, 1975.

JOSAI UNIVERSITY

SAKADO, SAITAMA 350-02, JAPAN 


\section{PACIFIC JOURNAL OF MATHEMATICS}

\section{EDITORS}

RICHARD ARENS (Managing Editor)

University of California

Los Angeles, California 90024

C. W. CURTIS

University of Oregon

Eugene, OR 97403

C. C. MOORE

University of California

Berkeley, CA 94720

\section{J. DugundJI}

Department of Mathematics University of Southern Californıa Los Angeles, California 90007

R. FinN AND J. Milgram Stanford University Stanford, California 94305

\section{ASSOCIATE EDITORS}

E. F. BECKENBACH

B. H. NEUMANN

F. WOLF

K. YOSHIDA

\section{SUPPORTING INSTITUTIONS}

UNIVERSITY OF BRITISH COLUMBIA CALIFORNIA INSTITUTE OF TECHNOLOGY UNIVERSITY OF CALIFORNIA MONTANA STATE UNIVERSITY UNIVERSITY OF NEVADA, RENO NEW MEXICO STATE UNIVERSITY OREGON STATE UNIVERSITY UNIVERSITY OF OREGON OSAKA UNIVERSITY
UNIVERSITY OF SOUTHERN CALIFORNIA STANFORD UNIVERSITY UNIVERSITY OF TOKYO UNIVERSITY OF UTAH WASHINGTON STATE UNIVERSITY UNIVERSITY OF WASHINGTON AMERICAN MATHEMATICAL SOCIETY NAVAL WEAPONS CENTER 


\section{Pacific Journal of Mathematics}

\section{Vol. 71, No. $1 \quad$ November, 1977}

Charalambos D. Aliprantis and Owen Sidney Burkinshaw, On universally complete Riesz spaces ............................. 1

Stephen Richard Bernfeld and Jagdish Chandra, Minimal and maximal solutions of nonlinear boundary value problems .................

John H. E. Cohn, The length of the period of the simple continued fraction of

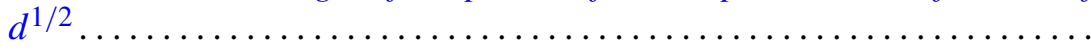

Earl Vern Dudley, Sidon sets associated with a closed subset of a compact abelian group .................................... 33

Larry Finkelstein, Finite groups with a standard component of type $J_{4} \ldots \ldots$

Louise Hay, Alfred Berry Manaster and Joseph Goeffrey Rosenstein, Concerning partial recursive similarity transformations of linearly ordered sets .......................................

Richard Michael Kane, On loop spaces without $p$ torsion. II ............

William A. Kirk and Rainald Schoneberg, Some results on

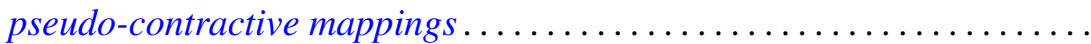

Philip A. Leonard and Kenneth S. Williams, The quadratic and quartic character of certain quadratic units. I. .

Lawrence Carlton Moore, A comparison of the relative uniform topology and the norm topology in a normed Riesz space .................

Mario Petrich, Maximal submonoids of the translational hull 119

Mark Bernard Ramras, Constructing new R-sequences . . .

Dave Riffelmacher, Multiplication alteration and related rigidity properties

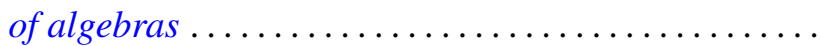

Jan Rosiński and Wojbor Woyczynski, Weakly orthogonally additive functionals, white noise integrals and linear Gaussian stochastic processes.

Ryōtarō Satō, Invariant measures for ergodic semigroups of operators

Peter John Slater and William Yslas Vélez, Permutations of the positive integers with restrictions on the sequence of differences...

Edith Twining Stevenson, Integral representations of algebraic cohomology classes on hypersurfaces ........................

Laif Swanson, Generators of factors of Bernoulli shifts . 